Mots. Les langages du politique

\title{
L'analyse du discours politique : de la théorie des champs à la sociologie de la grandeur
}

Political discourse sociology : from field theory to grandeur sociology

La sociología del discurso político : desde la teoria de los campos a la sociología de la grandeza

Christian Le Bart

\section{CpenEdition}

Journals

Édition électronique

URL : https://journals.openedition.org/mots/6323

DOI : $10.4000 /$ mots. 6323

ISSN : 1960-6001

Éditeur

ENS Éditions

Édition imprimée

Date de publication : 1 juillet 2003

Pagination : $97-110$

ISBN : 2-84788-034-8

ISSN : 0243-6450

Référence électronique

Christian Le Bart, «L'analyse du discours politique : de la théorie des champs à la sociologie de la grandeur », Mots. Les langages du politique [En ligne], 72 | 2003, mis en ligne le 29 avril 2008, consulté le 22 avril 2022. URL : http://journals.openedition.org/mots/6323; DOI : https://doi.org/10.4000/ mots. 6323 


\section{L'analyse du discours politique: de la théorie des champs à la sociologie de la grandeur}

La démocratie se caractérise en principe par la liberté laissée à chaque citoyen de prendre la parole pour donner son avis sur la chose publique. En pratique, parce qu'elle est indirecte et représentative, la démocratie tend à réserver l'accès à l'espace public aux professionnels de la politique, qui monopolisent de fait le droit de parler politique avec le privilège d'être entendus, pris au sérieux, commentés, voire obéis (Manin, 1995). La professionnalisation politique, en précipitant l'autonomisation du champ politique, a accentué la coupure entre représentants et représentés et, partant, la singularité du jeu politique.

Pièce essentielle de ce jeu, le discours politique, alors défini de façon restrictive comme le discours émanant des seuls acteurs investis dans le champ politique, apparait tout particulièrement marqué par cette singularité. Du point de vue des citoyens, il est perçu comme prévisible, codé, voire mensonger, plus intéressé qu'intéressant. Il suscite la méfiance. En termes plus scientifiques, on dira qu'il présente les caractéristiques d'un genre, avec ses contraintes, ses obligatoires et ses interdits. Langue de bois pour les uns, parole d'intérêt général pour les autres, le discours politique existe comme produit d'une activité discursive spécifique, il émane d'un champ social singulier. Il ne tire sa légitimité ni d'une quelconque prétention esthétique (encore que la rhétorique politique ait pu, par le passé, être rattachée à cette forme de grandeur), ni de sa prétention à décrire le monde tel qu'il est (encore que, là encore, le souci de réalisme ait pu y être intégré).

- CRAPE, Université Rennes 2 -Haute Bretagne, campus Villejean, 6, av. G. Berger, 35043 Rennes cedex, christian.lebart@Uhb.fr. 
Parce qu'il est le produit d'un champ social singulier (le champ politique), le discours politique doit donc être étudié comme genre singulier doté d'une certaine cohérence. Non que tous les énoncés produits dans (ou depuis) ce champ soient identiques, ou même simplement homologues, mais simplement parce que tous les énoncés politiques obéissent à des contraintes stables, que la sociologie du champ politique permet de mettre en lumière. Le discours politique ne reflète que superficiellement le vouloir-dire ponctuel d'un locuteur politique; encore moins reflète-t-il la vérité du monde social (même s'il arrive qu'un locuteur soucieux de paraitre dire vrai mobilise une rhétorique de la vraisemblance): le discours politique reflète l'état du champ politique au moment où il est produit ainsi que la position occupée, dans ce champ, par celui qui parle. Les remarques méthodologiques qui suivent se veulent les conséquences de cette hypothèse. Nous présentons ci-après une posture de recherche (parmi d'autres possibles) face à l'objet «discours politique», posture largement empruntée à la théorie des champs de Pierre Bourdieu, même si dans la dernière partie nous tenterons d'en identifier une limite en empruntant à la théorie de la grandeur proposée par Luc Boltanski et Laurent Thévenot.

\section{Approche structurale: l'obligation de souscrire aux illusios du champ politique}

Parler de champ pour désigner l'univers au sein duquel se déploie l'activité politique, c'est suggérer que les stratégies poursuivies par les acteurs politiques, les types de biens symboliques qu'ils produisent, qu'ils distribuent ou qu'ils convoitent, les comportements qu'ils adoptent, sont spécifiques à ce champ, et n'y prennent sens que relationnellement. L'autonomie du champ politique se marque dans la spécificité du capital qui prévaut en son sein (le capital politique), dans l'autonomie des institutions dites politiques, dans la division du travail qui consacre la singularité des acteurs politiques, dans l'originalité des règles du jeu politique (par exemple le suffrage universel), etc. Toutes ces dimensions demeureraient toutefois dépourvues de consistance si elles n'étaient adossées à un univers lui-même spécifique de croyances, croyances fondatrices de ce que Bourdieu nomme l'illusio. Un champ n'existe que s'il se trouve des croyances pour faire agir les acteurs, pour les motiver à acquérir le capital propre à celui-ci, ce qui suppose de croire en la valeur 
de ce capital et des trophées auxquels il permet d'accéder. "Au principe $\mathrm{du}$ fonctionnement de tous les champs sociaux (...), il y a l'illusio, l'investissement dans le jeu» (Bourdieu, 1998, p. 71). «L'illusio, c'est le fait d'être pris au jeu, d'être pris par le jeu, de croire que le jeu en vaut la chandelle» (Bourdieu, 1994, p. 151). Tous les acteurs d'un champ en partagent l'illusio, c'est ce consensus (souvent invisible de l'intérieur) qui rend les jeux internes au champ non seulement possibles mais même partiellement prévisibles.

Le terme illusio peut paraitre polémique: il vise simplement à rappeler que le chercheur ne peut comprendre les logiques d'un champ que depuis l'extérieur, c'est-à-dire en s'extrayant des croyances que partagent tous ceux qui sont dedans. L'explicitation puis l'analyse des croyances fondatrices d'un champ sont centrales pour qui veut comprendre par exemple les productions discursives internes à celui-ci. Car les discours sont déterminés, positivement ou négativement, par ces croyances: positivement, lorsque les locuteurs ravivent ces croyances; négativement, lorsque ces dernières bornent strictement les frontières (propres au champ politique) du dicible et même du pensable.

L'indiscuté politique, parce qu'il renvoie à ces croyances fondatrices de l'ordre politique, intéresse le chercheur alors qu'il n'intéresse pas les professionnels de la politique: ceux-ci l'ont intériorisé sous la forme d'évidences dont la remise en cause théorique entrainerait la remise en cause existentielle de leur rôle. De l'intérieur d'un champ, le comment l'emporte toujours sur le pourquoi, stérile car trop radical et trop menaçant. Certaines problématiques sont recevables dans le champ politique (comment accomplir au mieux son rôle d'élu? comment gouverner efficacement? etc.), d'autres pas, qui mettent en suspicion trop crument le champ politique (à quoi sert l'élection? pourquoi la démocratie doit-elle être représentative? etc.). L'entretien réflexif avec les professionnels de la politique ne permet guère, dans ces conditions, d'accéder directement aux croyances fondatrices du champ politique, car ceux-ci sont d'une certaine façon les moins bien placés pour en faire l'inventaire, prisonniers qu'il sont d'un rapport enchanté au politique. Elles apparaissent en revanche indirectement dans leurs discours, on l'a dit, au travers des évidences qui traversent ceux-ci et qui, parce qu'elles proviennent plus des impératifs du champ que du locuteur lui-même, passent à peu près inaperçus de tout le monde à l'intérieur.

Si les illusios sont invisibles pour ceux qui y adhèrent, c'est que ces derniers les ont fortement intériorisées, incorporées. L'illusio ne borne 
pas les frontières du politiquement dicible à la manière d'une contrainte externe comme le droit ou la surveillance de tous sur chacun. Auquel cas, elle s'apparenterait à une contrainte douloureuse parfaitement analysable par les intéressés. Elle est intériorisée sous la forme d'une façon d'être en politique par ceux qui ont été socialisés à croire en la politique et qui sont disposés à en faire. L'illusio prend la forme d'une foi ancrée qui, lorsqu'elle transforme une vocation en profession, apprend à faire l'économie d'une remise en cause. Il y a enchantement dans la mesure où on n'entre pas en politique sans croire en la politique comme activité noble, intéressante, ou au moins profitable. «L'adhésion fondamentale au jeu, l'illusio, [est] reconnaissance du jeu et de l'utilité du jeu, croyance dans la valeur du jeu et de son enjeu qui fondent toutes les donations de sens et de valeurs particulières » (Bourdieu, 1998, p. 288). Et si on discute, à l'intérieur du champ politique, des façons de faire de la politique, on apprend à ne plus y discuter de l'utilité-même d'un monde politique.

Une posture de recherche pertinente pour entrevoir les croyances génératrices de l'illusio politique est constituée par l'analyse des gaffes produites dans un champ. On appelle gaffe un énoncé qui, parce qu'il violente les croyances fondatrices du champ, suscite une réprobation collective de tous les acteurs du champ, voire l'autocritique contrite du locuteur. En politique, les gaffes révèlent les limites du politiquement dicible, et même sans doute du politiquement pensable. Elles disent, a contrario, ce sur quoi tous les entrants en politique doivent être d'accord, le consensus fondateur du champ politique.

Cette posture de recherche induit une conséquence quant à la délimitation des corpus. C'est à la marge que le discours politique révèle le mieux sa logique interne. Parce qu'ils sont déproblématisés, les énoncés ordinaires passent inaperçus car ils sont parfaitement conformes à l'horizon d'attente de ceux à qui ils sont destinés. En revanche, la dramatisation qui marque le franchissement des frontières dicible/indicible, recevable/irrecevable, permet la mise à nu des croyances qui sous-tendent le discours politique. Les périodes de routine, en ce sens, nous intéressent moins que les périodes de crise. Le scandale peut même constituer un indicateur fiable révélant une violence faite aux idéologies politiques fondatrices.

Il y a deux croyances fondatrices de l'illusio politique que les professionnels de ce secteur ne mettent jamais en discussion. Toutes deux participent de la légitimation du champ politique: la première en affirmant la grandeur originelle du pouvoir politique, par la sacralisation du suffrage universel; la seconde en affirmant sa grandeur fonctionnelle, par l'affir- 
mation de son efficacité à transformer la société. Depuis l'intérieur du champ politique, il est rigoureusement impossible de remettre en cause ces énoncés fondamentaux. Une parole hérétique qui affirmerait que le suffrage universel n'est rien, que les élus du peuple usurpent leur prétention à représenter le collectif, ou bien que de toutes façons le pouvoir est une comédie car l'Histoire se fait ailleurs qu'au sommet de l'État, cette parole hérétique est possible mais à l'extérieur du champ politique. Elle est impensable de l'intérieur. Car existe une solidarité minimale entre acteurs d'un même champ, ce que Bourdieu appelle «la collusion des agents dans l'illusio» (1998, p. 373).

La grandeur originelle du pouvoir politique repose sur la sacralisation du suffrage universel, véritable gisement de légitimité qui irrigue l'ensemble des institutions politiques démocratiques. En bénéficient des personnes (les élus du peuple ou de la nation), des rôles (parlementaire, maire, ministre...), des institutions, des textes, etc. Lorsque la légitimité du suffrage n'est qu'indirecte (Sénat, ou pire encore Conseil constitutionnel), la critique est possible de l'intérieur du champ politique, mais elle ne peut se déployer que dans le sens d'une exigence démocratique accrue. Le suffrage universel n'est jamais remis en cause en tant que tel, faire de la politique dans le contexte pacifié des démocraties représentatives suppose de s'incliner face à ses verdicts. Le peuple souverain est hors de portée de toute critique. Plus précisément, puisque les organes politiques tirent leur légitimité de leur capacité à incarner ce peuple, il ne saurait être question ni de remettre en cause cette prétention, ni même de mettre en discussion ces catégories de papier que constituent le peuple, la nation, la France, etc. Serait par exemple non exportable dans le champ politique le discours de la sociologie constructiviste selon lequel les communautés apparemment les plus évidentes comme les communautés nationales (mais on pourrait dire la même chose des jeunes, des femmes, des handicapés...) sont des catégories socialement construites, qui plus est si on ajoute qu'elles sont précisément construites par ceux qui prétendent les incarner. Dans le langage de la sociologie, c'est le représentant porteparole qui fait le groupe, dans celui de la politique, c'est le groupe qui se donne un représentant.

La grandeur fonctionnelle du pouvoir politique suppose la capacité à agir sur la société pour en régler les problèmes les plus aigus. L'hérésie consiste ici à dire (seconde variation sur le thème: le roi est nu) que le pouvoir politique tourne à vide, déploie sa magnificence en un pur spectacle qui vise au mieux à entretenir l'illusion d'un pouvoir efficace et 
bienveillant, au pire à masquer les vrais pouvoirs, ceux de la classe bourgeoise d'hier ou des multinationales d'aujourd'hui, ceux des hauts fonctionnaires et autres technocrates, etc. Ces discours désenchantés (auxquels là encore les sciences sociales, revendiquant une lucidité rendue possible par la position d'extériorité par rapport au champ politique, ne sont pas étrangères) ne sont pas pensables à l'intérieur du champ politique dans leur forme radicale. Ils heurtent trop violemment cette croyance sur laquelle toute vocation politique se fonde et qui veut qu'il soit possible d'agir sur le monde social depuis les positions de pouvoir offertes par les institutions politiques. La fatalité est structurellement interdite dans le champ politique. Cette conviction centrale entraine de multiples conséquences, dont par exemple la prétention des locuteurs politiques à toujours comprendre la société dans laquelle ils vivent, à toujours savoir donner du sens aux événements, à toujours pouvoir en identifier les causes. Si les professionnels de la politique souscrivent aujourd'hui volontiers à l'idée selon laquelle le monde social est complexe, s'ils soulignent plus que par le passé la nécessité de prendre le temps d'analyser et de comprendre, cet hommage apparent aux sciences sociales ne doit pas abuser: c'est en sociologues pressés qu'ils bricolent des causalités, qu'ils diagnostiquent des solutions ou qu'ils identifient des leviers d'action. Sommés du fait de leur position dans le champ politique de dire ce qu'il faut faire pour agir sur le monde, ils sont condamnés à entretenir le feu de la croyance en l'efficacité de l'action politique.

\section{Approche stratégique: les logiques de position dans le champ politique}

À la différence du chef indien observé par l'ethnologue Pierre Clastres (1974), les professionnels de la politique ne se contentent pas de répéter à l'infini le même discours obligé. Parler de discours contraint n'interdit pas d'identifier des stratégies ou des tactiques discursives liées à des intérêts ponctuels, personnels ou collectifs. Mais, parce que ces intérêts sont eux-mêmes internes au champ politique, les tactiques et stratégies doivent se déployer à l'intérieur de ces contraintes. Un locuteur les oublie-til, s'abandonnant à son seul intérêt du moment? Il devra essuyer la critique du champ politique dans son ensemble, amis politiques compris. C'est l'exemple des levées de boucliers suite aux gaffes de Lionel Jospin premier ministre qualifiant la droite française de «dreyfusarde», ou bien 
avouant son impuissance face aux licenciements chez Michelin. C'était oublier que la démocratie représentative interdit la disqualification de l'adversaire lorsqu'il bénéficie de la légitimité du suffrage universel, c'était oublier qu'un gouvernement ne peut faire publiquement le constat de son impuissance face à un problème social.

Si toute prise de parole dans le champ politique s'analyse donc comme variation autour des quelques énoncés fondamentaux que l'on vient d'identifier, chaque locuteur a sa façon bien à lui d'exécuter cette partition commune à tous. Importe moins ici le style personnel (encore que celui-ci, comme produit d'une trajectoire de socialisation individuelle, puisse compter) que la position objective occupée par le locuteur dans le champ politique et la structure singulière d'intérêts (politiques) que cette position conditionne. Tout discours politique doit donc s'analyser à la fois structurellement comme variation autour des croyances fondatrices de l'illusio politique et stratégiquement comme reflet de la position relative occupée par le locuteur ${ }^{1}$. Dans les deux cas, les logiques de champ tendent à supplanter les déterminations externes, par exemple par référence aux attentes des électeurs :

Dire qu'il y a un champ politique, c'est rappeler que les gens qui s'y trouvent peuvent dire ou faire des choses qui sont déterminées non par la relation directe avec les votants, mais avec les autres membres du champ. [Un acteur politique] dit ce qu'il dit [...] non pas pour répondre aux attentes de la population en général ou même de la catégorie qui lui a donné sa voix, qui l'a désigné comme mandataire, mais par référence à ce que d'autres dans le champ disent ou ne disent pas, font ou ne font pas (Bourdieu, 2000, p. 57).

L'énoncé percutant est ainsi celui qui vient au bon moment, dans la bouche d'un locuteur autorisé, et qui conjugue idéalement l'intérêt politique de celui qui parle et le respect scrupuleux des illusios que partagent ceux qui écoutent. Concrètement, chaque rôle politique renvoie à une modalité originale d'agencement des énoncés fondamentaux précédents. Jouer son rôle de ministre, de leader de l'opposition, de responsable d'un parti de gouvernement, ou de président de la République, c'est s'inscrire dans un sous-ensemble relativement rigide de prescriptions qui encadrent précisément les prises de parole. Le clivage entre gouvernants et oppo-

1. Cette seconde posture suppose la construction de corpus cohérents par référence à un rôle. Ainsi analysera-t-on le discours des maires, des sénateurs, des ministres, des chefs de partis de l'opposition, des candidats en campagne, des femmes de présidents, etc. 
sants est sans doute central: non que les seconds soient, comme on le croit trop souvent, libres de leur parole quand les premiers seraient engoncés dans des rôles fermés. Ce clivage est pertinent tout simplement parce qu'il renvoie à deux façons de décliner les fondamentaux du champ politique. Côté gouvernants : en rappelant sa propre légitimité et en assumant la prétention à agir sur la société (promesse d'action pour l'avenir, mise en scène décisionnelle, effet d'annonce et politique symbolique pour le présent, discours d'auto-imputation pour le passé). Côté opposants : en développant une critique des gouvernants qui ne soit jamais une critique du rôle qu'ils occupent (la fonction est noble, mais son titulaire n'est pas à la hauteur); et en dénonçant une impuissance qui là encore est coupable et non structurelle (version 1 : ils sont au pouvoir et ils ne font rien; version 2: ils poursuivent efficacement un programme nuisible).

Gouvernants et opposants ne disent pas la même chose, mais les argumentaires différenciés et apparemment contradictoires qu'ils développent empruntent à un même univers d'illusios. Cet inconscient du discours politique est le socle indépassable à partir duquel la parole politique se déploie. Il est exceptionnel que cet inconscient donne lieu à explicitation pure et simple, parce qu'il est exceptionnel que la stratégie discursive d'un locuteur s'y réduise. Une telle situation se rencontre en revanche lorsqu'un politique sort du champ politique, par exemple s'il s'adresse à des individus qui lui apparaissent très éloignés du modèle citoyen. Alors prendra-t-il en charge la défense de l'ensemble du champ politique, par exemple face à des enfants pour rappeler ce qu'est la démocratie et ce que permet l'exercice du pouvoir politique. Plus souvent, ces énoncés fondamentaux sont, comme on l'a dit, instrumentalisés pour servir une argumentation interne au champ politique.

Est-ce à dire qu'il est impossible de trouver trace, dans le champ politique, donc dans le discours (des professionnels de la) politique, de remises en cause des croyances fondatrices de l'illusio politique? Les choses ne sont pas aussi simples: un champ social n'est pas seulement caractérisé par l'opposition entre dedans (ceux qui y croient et dont la parole est chargée d'illusio) et dehors (ceux qui peuvent ne pas y croire et qui disent ce qu'ils veulent). Le champ politique est aussi structuré sur la base d'une opposition entre positions dominantes et positions dominées. Comment situer, dans le schéma précédent, les non-professionnels de la politique, les 500000 conseillers municipaux, les «simples» militants, ou bien encore les responsables de tout petits partis jamais élus au suffrage universel parce que extrémistes ou ultra-minoritaires? 
Côté professionnels de la politique, les choses sont simples : l'abandon de la liberté de parole conditionne l'exercice du pouvoir. Le discours d'un président de la République est à la fois très contraint (il est prévisible car très ritualisé) et très puissant (beaucoup des énoncés présidentiels sont chargés d'une forte performativité: ainsi lorsque le président décide, appelle, convoque, dissout, nomme, approuve ou désapprouve au nom de la France, etc.). Mais que dire des locuteurs en position inférieure ou latérale? Moins surveillés, moins écoutés, ils peuvent davantage s'autoriser quelques libertés par rapport aux croyances fondatrices du champ politique. Mais ces libertés, parce qu'on reste à l'intérieur du champ en question, ne vont jamais jusqu'à l'hérésie pure. Comme les autres locuteurs, les acteurs dominés du champ politique doivent couler leur propre message (c'est-à-dire développer leur propre stratégie) dans la double célébration (originelle et fonctionnelle) du pouvoir politique. Par exemple, un petit élu (le maire d'une commune rurale) peut certes dénoncer la crise de la représentation qui selon lui coupe les «politiciens» de la réalité de la vie quotidienne. Son apologie d'une proximité qu'il prétend, lui, incarner, vaut critique de la représentation politique. Mais cette critique ne va pas jusqu'à devenir de principe, auquel cas il serait lui-même emporté. Il demeure partisan de la délégation qui fonde la démocratie représentative, même s'il plaide pour un rapprochement entre professionnels de la politique et simples citoyens. Ce discours d'auto-légitimation n'est pas hérétique. Même chose à la périphérie du champ politique, lorsqu'on dénonce l'impuissance (coupable ou intentionnelle) des gouvernants. Le discours apparemment le plus radical, de l'extrême droite à l'extrême gauche, emprunte en réalité à une conception très conformiste du pouvoir politique comme pouvoir efficace qui doit permettre de changer la société $s i$ on le veut vraiment.

\section{Approche dynamique : l'importation de grandeurs alternatives}

La théorie des champs de Pierre Bourdieu, à laquelle nous empruntons la démarche précédente, achoppe, au moins dans le cas du champ politique, sur une difficulté redoutable: ce champ n'est pas fermé sur lui-même. Le suffrage universel, capital singulier spécifique à cet univers, permet l'accès aux positions dominantes dans le champ, mais il 
résulte d'un échange avec des acteurs extérieurs à celui-ci². Les qualifier d'électeurs ou de citoyens ne change rien à la chose: le pouvoir en politique trouve sa source loin du petit monde des professionnels de la politique. Cette donnée fondamentale suffit à distinguer le champ politique de champs dont l'autonomie est parfois synonyme de clôture (monde universitaire ou monde des avant-gardes artistiques, par exemple, également indifférents voire hostiles à l'approbation des individus hors champ ${ }^{3}$ ). Pierre Bourdieu, comparant sur ce point le champ politique au champ religieux, en convient d'ailleurs :

Le champ politique a une particularité: il ne peut jamais s'autonomiser complètement. Il est sans cesse référé à sa clientèle, aux laïcs, et ces laïcs ont en quelque sorte le dernier mot dans les luttes entre clercs, entre membres du champ (Bourdieu, 2000, p. 62-63).

Si autonomie du champ politique il y a, celle-ci est donc éminemment relative. Sur le terrain qui nous intéresse, celui du discours politique, cette tension entre autonomie et hétéronomie se marque dans la juxtaposition de deux familles d'énoncés: d'une part les énoncés fondateurs du champ politique qui ont jusqu'à présent retenu notre attention, d'autre part des énoncés puisant dans des registres de légitimation extérieurs au champ politique. Les mécanismes d'importation, vers le champ politique, de grandeurs alternatives à la grandeur politique classique, sont d'autant plus visibles aujourd'hui que la grandeur strictement politique tend à s'essouffler. La légitimité politique, qu'elle soit fondée sur l'invocation du suffrage universel, ou sur la prétention à agir sur la société, fait certes toujours l'objet d'appels incantatoires, mais on a l'impression que ces croyances perdent une partie de leur poids dès qu'on s'éloigne du champ politique. La crise de la représentation est peut-être là, dans l'impossibilité pour les hommes politiques de transformer les croyances fondatrices du champ politique en croyances partagées par tous, en ressources de légitimité externes; autrement dit d'intégrer au champ politique les simples individus, d'en faire des citoyens acceptant de reconnaitre la

2. L'électeur est-il ou non dans le champ politique ? Voir les remarques de Bernard Lahire, « Champ, hors-champ, contre-champ» (B. Lahire, 2001).

3. On peut être un grand universitaire en écrivant des livres qui ne se vendent pas, un grand peintre en produisant une œuvre à laquelle le grand public ne comprend rien, mais peut-on être un grand politique sans la reconnaissance du suffrage universel ? 
valeur d'un champ auquel ils n'appartiennent pas directement, mais qui ne peut se reproduire sans leur consentement.

Puisque les professionnels de la politique ne parviennent plus guère à exporter et à universaliser les formes de grandeur spécifiquement politiques, ils ne peuvent maintenir le contact avec la société civile qu'en important à l'inverse des grandeurs ayant cours au sein de celle-ci, pourvu qu'elles soient à peu près compatibles avec la leur. C'est ainsi qu'on peut lire la transformation contemporaine du champ politique dans le sens d'une plus grande personnalisation. Auparavant, le titulaire d'un rôle politique pouvait se satisfaire de la légitimité conférée à celui-ci: grandeur du suffrage universel, grandeur de la fonction de décideur-gouvernant. Ces deux grandeurs demeurent, mais tout se passe comme si elles ne suffisaient plus. D'où la stratégie qui consiste à légitimer le titulaire du rôle pour pallier l'essoufflement de la grandeur du rôle lui-même. Ainsi voit-on les professionnels de la politique conduire des stratégies de légitimation personnelle, au double sens de l'expression: elle leur profite personnellement (quand la grandeur politique classique était octroyée collectivement à travers des rôles, des institutions), elle repose sur la seule mise en scène de leur personne. La montée en puissance de la thématique de l'intime, que l'on peut analyser comme féminisation de la vie politique, exprime un bouleversement de fond: ce qui jadis devait être caché afin que la légitimation par le rôle puisse s'effectuer pleinement, est aujourd'hui mis en avant ${ }^{4}$. Lorsque le rôle suffisait à légitimer, on pouvait (on devait) se réfugier derrière lui. L'effritement de celui-ci oblige à œuvrer à la grandeur du titulaire du rôle, dès lors que celui-ci a fini d'être grand par sa seule force intrinsèque. En terme wébérien, on peut dire que les politiques sont condamnés au charisme. En terme éliasiens, qu'ils peuvent sans réserve emprunter à la nostalgie chevaleresque, c'est-à-dire tirer argument de leur incapacité à contrôler parfaitement leurs émotions et à faire taire leur intériorité. À l'extrême la légitimité peut être revendiquée, jusque dans le champ politique, sans référence au politique. Ainsi voit-on des professionnels de la politique se présenter comme ne faisant pas de politique ${ }^{5}$, ou publier des confidences pour dire

4. Voir les travaux d'Érik Neveu (par exemple 1995) ou le récent numéro de Mots sur «La politique à l'écran: l'échec» $\left(n^{\circ} 67,2001\right)$.

5. «Moi je ne fais pas de politique, je ne suis pas un politique» (Jean Saint-Josse, candidat à l'élection présidentielle, Libération, 19 avril 2002) 
leur peu de gout pour la politique. Ces entreprises individuelles, une fois agglomérées, fragilisent un peu plus la grandeur spécifiquement politique. On peut se demander ce qu'il reste, dans un tel contexte, des légitimations traditionnelles du politique que nous avons tenté d'identifier plus haut. Elles demeurent sans doute, ce qui suffit à rendre pertinente encore aujourd'hui la théorie des champs dans ce qu'elle peut avoir de structurale et d'a-historique. Mais ces croyances fondatrices de l'illusio s'accommodent de discours de légitimation alternatifs, avec lesquels elles sont certes compatibles, mais qui tendent à éclipser leur centralité passée. La grandeur en politique est de moins en moins la grandeur politique.

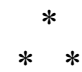

C'est par exemple ce que démontre le statut aujourd'hui conféré aux coulisses de la vie politique ${ }^{6}$. Vie privée, personnalité «vraie», loisirs et hobbies, petites phrases prononcées off, moments de relâchement... : tout ceci intéressait jadis les journalistes attachés à dévoiler une part de vérité par-delà les apparences institutionnelles. Derrière la langue de bois, on traquait la petite phrase libérée des illusios, et censée dévoiler la vérité crue du jeu politique. À présent, il est trop évident que les coulisses sont elles-mêmes mises en scène par les professionnels de la politique qui travaillent leur présentation de soi dans deux contextes complémentaires mais distincts: sur la scène officielle, là où il faut tenir son rôle, et dans des coulisses désormais quasi publiques, là où s'acquièrent la légitimité que le seul rôle ne suffit plus à conférer. Cet exemple montre que les illusios politiques ont perdu leur centralité dans le le champ politique. Elles ne constituent plus qu'un dispositif de légitimation parmi d'autres. À l'horizon de ce jeu d'hypothèses, on voit se substituer au modèle structural du champ politique un modèle beaucoup plus volatil, celui des mondes, des grandeurs, des cités de Luc Boltanski et Laurent Thévenot. Des gisements de légitimité existent dans lesquels les professionnels de la politique vont ouvertement puiser. La grandeur civique n'est plus, dans le

6. On pense en particulier aux films sur les campagnes électorales, depuis celle de VGE en 1974 (Depardon) jusqu'à la campagne parisienne de 2001(Moati et Jeuland). Le succès de ces entreprises de «dévoilement» est à rapprocher du déclin des émissions politiques classiques, comme si le regard du public s'était déplacé de la scène vers les coulisses. 
champ politique y compris, qu'une grandeur parmi d'autres. Et le champ politique perd sans doute, au gré de ces mutations, une part de son autonomie. D'où une incertitude croissante quant aux délimitations de corpus: les professionnels de la politique cherchent à parfaire leur légitimité politique en acceptant de parler publiquement d'autre chose que de politique. Les frontières de l'objet «discours politique» sont devenues mouvantes.

\section{Références bibliographiques}

BOLTANSKI L., CHIAPELLO É., 1999, Le nouvel esprit du capitalisme, Paris, Gallimard.

BOLTANSKI L., THÉVENOT L., 1991, De la justification, les économies de la grandeur, Paris, Gallimard.

BOURDIEU P., 1998, Les règles de l'art, genèse et structure du champ littéraire, Paris, Le Seuil.

BOURDIEU P., 2000, Propos sur le champ politique, Lyon, Presses universitaires de Lyon.

BOURDIEU P., 1994, Raisons pratiques. Sur la théorie de l'action, Paris, Le Seuil.

CLASTRES P., 1974, La société contre l'État, Paris, Minuit.

LAHIRE B. (dir.), 2001, Le travail sociologique de Pierre Bourdieu, dettes et critiques, Paris, La Découverte.

LE BART C., 2001, Le discours politique, Paris, PUF, «Que sais-je?».

MANIN B., 1995, Principes du gouvernement représentatif, Paris, CalmannLevy.

Mots, décembre 2001, n 67, La politique à l'écran: l'échec?

NEVEU É., 1995, «Les émissions politiques à la télévision: les années 1980 ou les impasses du spectacle politique», Hermès, $\mathrm{n}^{\circ}$ 17-18, p. 145-162. 